\title{
KAEHLER MANIFOLDS OF POSITIVE CURVATURE OPERATOR
}

\author{
KOICHI OGIUE AND SHUN-ICHI TACHIBANA
}

\begin{abstract}
An n-dimensional compact Kaehler manifold of positive curvature operator is real cohomologically equivalent to $P_{n}(C)$.
\end{abstract}

1. Introduction. The curvature tensor of a Riemannian manifold defines a symmetric linear operator acting on the space of 2-forms, which is called the curvature operator. D. Meyer [3] proved that a compact Riemannian manifold of positive curvature operator is a real homology sphere.

The purpose of this paper is to study the complex version of Meyer's result. It is known that the curvature tensor of a Kaehler manifold defines two kinds of linear operators. Let $M$ be a Kaehler manifold and $R_{\bar{a} b c \bar{d}}$ the local components of the curvature tensor of $M$. The operator acting on symmetric 2-tensors defined by $\xi_{a b} \rightarrow \sum R_{b c}^{a}{ }_{b a d}$ is called the pure curvature operator, and the operator acting on 2 -forms defined by $\eta_{a \bar{b}} \rightarrow \sum R^{a \bar{b}}{ }_{c \bar{d}} \eta_{a \bar{b}}$ is called the hybrid curvature operator. These curvature operators are said to be positive if all eigenvalues are positive. It is clear that if one of the curvature operators is positive, then so is the sectional curvature. The properties of the pure curvature operator for Hermitian symmetric spaces were precisely studied by E. Calabi [1].

We shall prove the following.

THEOREM 1. An n-dimensional compact Kaehler manifold of positive pure curvature operator is real cohomologically equivalent to $P_{n}(C)$.

TheOREM 2. An n-dimensional compact Kaehler manifold of positive hybrid curvature operator is real cohomologically equivalent to $P_{n}(C)$.

Theorem 2 can be considered as an improvement of Theorem 2.3 in [4].

2. Proof of theorems. Let $\boldsymbol{M}$ be an $\boldsymbol{n}$-dimensional compact Kaehler manifold. We shall make use of the following convention on the range of indices: $1<a, b, c, d$ $<n ; 1<i, j, k, l \leqslant 2 n$, and we shall agree that repeated indices are summed over the respective ranges unless otherwise stated.

Let $R_{i j k l}$ (resp. $R_{\bar{a} b c \bar{d}}$ ) and $R_{i j}$ (resp. $R_{a \bar{b}}$ ) denote respectively the local components of the curvature tensor and the Ricci tensor of $M$ with respect to a real (resp. complex) local coordinate system. We use the same curvature tensor as in [2]. To prove our theorems, it is sufficient to show that

Received by the editors November 16, 1978 and, in revised form, April 25, 1979.

AMS (MOS) subject classifications (1970). Primary 53C55.

Key words and phrases. Kaehler manifold, curvature operator, cohomology, $P_{n}(C)$.

(C) 1980 American Mathematical Society 0002-9939/80/0000-0169/\$01.75 


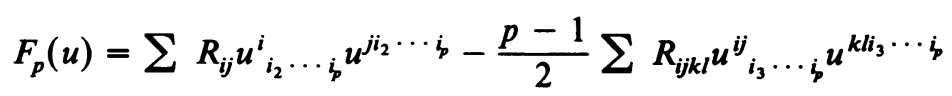

is positive for nonzero effective harmonic $p$-form $u$ (cf. for example [5]. Note that our curvature tensor is the negative of the one in [5]). it is easily seen that

$$
F_{p}(u)=2\left\{\sum R_{a \bar{b}} u^{a}{ }_{i_{2}} \cdots_{i_{p}} u^{\bar{b} i_{2} \cdots i_{p}}-(p-1) \sum R_{\bar{a} b c \bar{d}} u^{\bar{a} b}{ }_{i_{3} \cdots j_{p}} u^{c \bar{d} i_{3} \cdots i_{p}}\right\} .
$$

Suppose that the pure curvature operator is positive. Let $u$ be a nonzero effective harmonic $p$-form and define a $(p+2)$-tensor $\xi$ by

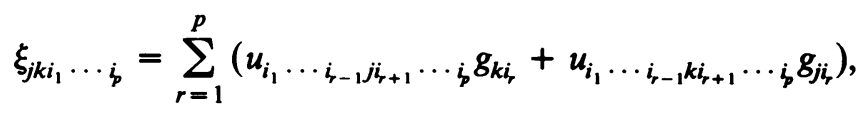

where $g_{i j}$ are the local components of the Kaehler metric. Then it follows that

$$
\sum R_{b c}^{a}{ }^{d} \xi_{a d i_{1}} \cdots i_{p} \xi^{b c i_{1} \cdots i_{p}}=2 p F_{p}(u)
$$

On the other hand, we see that

$$
\begin{aligned}
\sum \xi_{a b i_{1} \cdots j_{p}} \xi^{a b i_{1} \cdots i_{p}}= & p\left\{(n+1)\|u\|^{2}-2(p-1) \sum u_{a \bar{b} i_{3} \cdots j_{p}} u^{a \overline{b_{3}} \cdots i_{p}}\right\} \\
\geqslant p\left\{(n+1)\|u\|^{2}-(p-1) \sum u_{a \overline{b i} i_{3} \cdots j_{p}} u^{a \overline{b i_{3}} \cdots i_{p}}\right. & \left.\quad-(p-1)\left(\frac{1}{2}\|u\|^{2}+\sum u_{a b i_{3} \cdots j_{p}} u^{a b i_{3} \cdots i_{p}}\right)\right\} \\
= & p(n-p+2)\|u\|^{2},
\end{aligned}
$$

where we have used the simple inequality

$$
\|u\|^{2}=\sum u_{i_{1} \cdots i_{p}} u^{i_{1} \cdots i_{p}} \geqslant 2 \sum\left(u_{a \bar{b} i_{3} \cdots i_{p}} u^{a \overline{b i} i_{3} \cdots i_{p}}-u_{a b i_{3} \cdots i_{p}} u^{a b i_{3} \cdots i_{p}}\right) .
$$

Since the pure curvature operator is positive, there exists a positive number $\delta$ such that

$$
\sum R_{b c}^{a}{ }^{d} \xi_{a d i_{1} \cdots i_{p}} \xi^{b c i_{1} \cdots i_{p}} \geqslant \delta \sum \xi_{a b i_{1} \cdots i_{p}} \xi^{a b i_{1} \cdots i_{p}} \text {, }
$$

that is, $2 F_{p}(u) \geqslant \delta(n-p+2)\|u\|^{2}$. This implies that $F_{p}(u)>0$ for nonzero effective harmonic $p$-form $u$ for $p<n+2$. Therefore there exists no nonzero effective harmonic $p$-form for $p<n+2$ and hence $M$ is real cohomologically equivalent to $P_{n}(C)$, which proves Theorem 1 .

Suppose next that the hybrid curvature operator is positive. Let $u$ be a nonzero effective harmonic $p$-form and define a $(p+2)$-tensor $\eta$ by

$$
\eta_{j k i_{1} \cdots i_{p}}=\sum_{r=1}^{p}\left(u_{i_{1} \cdots i_{r-1} j i_{r+1} \cdots i_{p}} g_{k i_{r}}-u_{i_{1} \cdots i_{r-1} k i_{r+1} \cdots j_{p}} g_{j i_{r}}\right) \text {. }
$$

Then it follows that

$$
\sum R_{c \bar{b}}^{a \bar{b}} \eta_{a \bar{b} i_{1}} \ldots j_{p} \eta^{c \bar{d} \bar{d}_{1} \cdots i_{p}}=p F_{p}(u)
$$


On the other hand, we see that

$$
\begin{aligned}
& \sum \eta_{a \bar{b} i_{1}} \cdots_{j} \eta^{a \overline{b i}_{1} \cdots j_{p}}=p\left\{n\|u\|^{2}-2(p-1) \sum u_{a b i_{3} \cdots j_{p}} u^{a b i_{3} \cdots \dot{j}_{p}}\right\} \\
& \geqslant p\left\{n\|u\|^{2}-(p-1) \sum u_{a b i_{3} \cdots i_{p}} u^{a b i_{3} \cdots i_{p}}\right. \\
& \left.-(p-1)\left(\frac{1}{2}\|u\|^{2}+\sum u_{a \bar{b} i_{3}} \cdots_{j} u^{a \bar{b} i_{3} \cdots j_{p}}\right)\right\} \\
& =p(n-p+1)\|u\|^{2},
\end{aligned}
$$

where we have used the simple inequality

$$
\|u\|^{2}=\sum u_{i_{1} \cdots j_{p}} u^{i_{1} \cdots i_{p}} \geqslant 2 \sum\left(u_{a b i_{3} \cdots j_{p}} u^{a b i_{3} \cdots i_{p}}-u_{a \bar{b} i_{3} \cdots i_{p}} u^{a \bar{b} i_{3} \cdots i_{p}}\right) .
$$

Since the hybrid curvature operator is positive, there exists a positive number $\delta$ such that

$$
\sum R^{a \bar{b}}{ }_{c \bar{d}} \eta_{a \bar{b} i_{1} \cdots i_{p}} \eta^{c \bar{d} \bar{i}_{1} \cdots i_{p}} \geqslant \delta \sum \eta_{a \bar{b} i_{1} \cdots i_{p}} \eta^{a \bar{b} i_{1} \cdots i_{p}},
$$

that is, $F_{p}(u)>\delta(n-p+1)\|u\|^{2}$. This implies that $F_{p}(u)>0$ for nonzero effective harmonic $p$-form $u$ for $p<n+1$. Therefore there exists no nonzero effective harmonic $p$-form for $p<n+1$ and hence $M$ is real cohomologically equivalent to $P_{n}(C)$, which proves Theorem 2 .

\section{REFERENCES}

1. E. Calabi and E. Vesentini, On compact locally symmetric Kaehler manifolds, Ann. of Math. 71 (1960), 472-507.

2. S. Kobayashi and K. Nomizu, Foundations of differential geometry. II, Interscience, New York, 1969.

3. D. Meyer, Sur les variétés riemanniennes à opérateur de courbure positif, C. R. Acad. Sci. Paris Sér. A-B 272 (1971), 482-485.

4. S. Tachibana, On Kaehlerian manifolds of o-positive curvature operator, Natur. Sci. Rep. Ochanomizu Univ. 25 (1974), 7-16.

5. K. Yano and S. Bochner, Curvature and Betti numbers, Ann. of Math. Studies, no. 32, Princeton Univ. Press, Princeton, 1953.

Department of Mathematics, Tokyo Metropolttan University, Setagaya, Tokyo, 158 Japan

Department of Mathematics, Ochanomizu University, Otsuka, Bunkyo-Ku, Tokyo 112, Japan 